\title{
FOURIER ANALYSIS OF NONSTATIONARY STOCHASTIC PROCESSES ${ }^{(1)}$
}

\author{
BY \\ TATSUO KAWATA
}

1. Introduction. In the present paper we shall deal with the Fourier integral theory of the general stochastic processes. A general theory of covariance functions of a nonstationary process was developed by M. Loève [8], [9] and some detailed discussions were also given by him on a special process called the harmoniable process, in which he covered the harmonic representation theorem. A number of papers dealing with nonstationary processes appeared in connection with noise theory or the statistical theory of regression (see E. Parzen [10]). H. Cramér [4] extended the criterion concerning purely indeterministic stationary processes to the case of harmonisable processes and also gave a decomposition theorem.

Still, it seems to me that very few papers, except those on the very general mathematical structure like measurability, separability, etc., were devoted to the study of general nonstationary processes.

The aim of this paper is to develop the Fourier integral theory of the general nonstationary process $X(x, \omega)$. Usually $x$ is taken as a parameter which represents time, but in this paper we do not have to emphasize the time character of the process. $\omega$ represents an element of the given probability field $\Omega$.

In fact, we shall prove some theorems which are analogous to those in the ordinary $L_{1}$ theory of Fourier transform, such as summability theorems, inversion formulas for Fourier transforms and the Wiener formula.

It will be of interest to notice that these theorems have some meaning from a probabilistic point of view. For instance, the summability theorems concerning stochastic processes will be thought of as the smoothing or filtering of these processes; the Wiener formula will turn out to be some type of law of large numbers and the inversion formula for Fourier transforms will give the harmonic representation for the process.

We shall suppose throughout this paper that the stochastic process $X(x, \omega)$, $-\infty<x<\infty, \omega \in \Omega$, being a probability field, satisfies the conditions:

(i) it is measurable and separable,

(ii) $E|X(x, \omega)|^{2}<\infty$ for every $x$,

(iii) $\int_{a}^{b} E|X(x, \omega)|^{2} d x<\infty$ for every finite interval $(a, b)$,

(iv) $E X(x, \omega)=0$ for every $x$ and

Received by the editors January 16, 1964.

(1) This work was supported by NSF Grant, GP-1577. 
(v) the covariance function

$$
\rho(s, t)=E X(s, \omega) \overline{X(t, \omega)}
$$

is continuous in $-\infty<s, t<\infty$.

Because of (iii), $X(x, \omega)$ is a function of $L_{2}$ over every finite $x$-interval with probability 1 . Also $X(x, \omega)$ is continuous in the $L_{2}$-mean for every $x$, that is,

$$
\lim _{h \rightarrow 0} E|X(x+h, \omega)-X(x, \omega)|^{2}=0 .
$$

The condition (i) may be deleted based on the continuity condition (1.2) if a measurable and separable modification of $X(x, \omega)$ is adopted (Doob [5, Chapter 2]).

The integral $\int_{a}^{b} X(x, \omega) d x$ over a finite interval $(a, b)$ is defined in view of (iii). The integral of $X(x, \omega)$ over $(-\infty, \infty)$ is defined as $X_{1}(\omega)$, if

$$
E\left|\int_{A}^{B} X(x, \omega) d x-X_{1}(\omega)\right|^{2} \rightarrow 0
$$

as $A \rightarrow-\infty, B \rightarrow \infty$. $X_{1}(\omega)$ is uniquely determined with probability 1 and is described as

$$
X_{1}(\omega)=\lim _{A \rightarrow-\infty, B \rightarrow \infty} \int_{A}^{B} X(x, \omega) d x
$$

or simply

If we suppose that

$$
X_{1}(\omega)=\int_{-\infty}^{\infty} X(x, \omega) d x
$$

$$
\iint_{-\infty}^{\infty}|\rho(s, t)| d s d t<\infty,
$$

we may define the Fourier transform $Y(y, \omega)$ of $X(x, \omega)$ by

$$
\frac{1}{\sqrt{ }(2 \pi)} \int_{-\infty}^{\infty} e^{-i y x} X(x, \omega) d x .
$$

It is easily seen that $Y(y, \omega)$ is continuous for every $y$ and

$$
\underset{|y| \rightarrow \infty}{\operatorname{lim.m} \mid} Y(y, \omega)=0,
$$

because

$$
\begin{aligned}
& E|Y(y+h, \omega)-Y(y)|^{2}=\lim _{A \rightarrow-\infty, B \rightarrow \infty} E\left|\frac{1}{\sqrt{ }(2 \pi)} \int_{A}^{B} e^{-\imath y x}\left(e^{-i h x}-1\right) X(x, \omega) d x\right|^{2} \\
& =\frac{1}{2 \pi} \iint_{-\infty}^{\infty} e^{-i y(s-t)}\left(e^{-i h s}-1\right)\left(e^{-i h t}-1\right) \rho(s, t) d s d t
\end{aligned}
$$

which converges to zero as $h \rightarrow 0$. 
The proof of (1.7) is also quite easy.

The Fourier transform $Y(y, \omega)$ is defined with probability 1 for every $y$; the exceptional set of probability 0 may depend on $y$. Still the Fourier transform may be thought of as a uniquely defined function of $y$ with probability 1 , if the separable and measurable modification is taken for $Y(y, \omega)$; this is possible because $Y(y, \omega)$ is continuous.

We shall agree to make this convention when it is necessary. The condition (1.6) which enables us to define Fourier transforms, is, however, inappropriate in many cases. Spectral analysis with this definition of Fourier transform does not seem adequate except in some specific cases.

While we shall give some theorems on the Fourier transform above-defined, we shall consider the generalization of the Fourier transform. As a matter of fact, we shall give the generalization of the $k$-transform to the stochastic case. Particular attention is paid to the 2-transform in which we have only to impose the condition

$$
\iint_{-\infty}^{\infty} \frac{|\rho(s, t)|}{\left(1+s^{2}\right)\left(1+t^{2}\right)} d s d t<\infty
$$

The $k$-transform was defined by S. Bochner [2] and is now classical and well known. The harmonic representation theorem will be given in $\$ 6$ by using this type of generalized transform.

2. Some elementary lemmas. It will be convenient to state a few lemmas that are quite simple and well known, but which are of frequent use in the subsequent discussions.

LEMMA 2.1. If the covariance function $\rho(s, t)$ of a stochastic process $X(x, \omega)$ satisfying (i), (ii), (iii) and (iv) in $§ 1$, satisfies the condition that

$$
\iint_{-\infty}^{\infty}\left|\rho(s, t) f_{k}^{2}(s)\right| d s d t<\infty, \quad \iint_{-\infty}^{\infty}\left|\rho(s, t) f_{k}^{2}(t)\right| d s d t<\infty, k=1,2
$$

for some numerical functions $f_{1}(t)$ and $f_{2}(t)$, then the integral

$$
Y_{k}(\omega)=\int_{-\infty}^{\infty} X(x, \omega) f_{k}(x) d x, \quad k=1.2
$$

exists and

$$
E Y_{1}(\omega) \overline{Y_{2}(\omega)}=\iint_{-\infty}^{\infty} \rho(s, t) f_{1}(s) \overline{f_{2}(t)} d s d t
$$

This was already used in deriving (1.9).

In particular, if $f_{k}(t)=e^{-i t y} / \sqrt{ }(2 \pi)(k=1,2, y$ being a parameter $)$, then $Y_{k}(\omega)$ will be the Fourier transform of $X(x, \omega)$, 


$$
\frac{1}{\sqrt{(2 \pi)}} \int_{-\infty}^{\infty} X(x, \omega) e^{-i x y} d x .
$$

This has been defined in $\S 1$ under the condition (1.6).

LEMMA 2.2. If $f(x)$ is a numerical function of $L_{2}(a, b),-\infty<a<b<\infty$ an $a$ the process $X_{A}(x, \omega)$ which depends on a parameter $A$ is such that

$$
\underset{A \rightarrow \infty}{\lim .} X_{A}(x, \omega)=X(x, \omega)
$$

for almost every $x$ in $(a, b)$ and

$$
E\left|X_{A}(x, \omega)\right|^{2}<M,
$$

$M$ being a constant independent of $A$ and $x$, then

$$
\underset{A \rightarrow \infty}{\lim . \log } \int_{a}^{b} f(x) X_{A}(x, \omega) d x=\int_{a}^{b} f(x) X(x, \omega) d x .
$$

This is immediate in view of Schwarz's inequality, because

$$
\begin{array}{rl}
E \mid \int_{a}^{b} f(x) X_{A}(x, \omega) d x-\int_{a}^{b} & \left.f(x) X(x, \omega) d x\right|^{2} \\
& \leqq \int_{a}^{b}|f(x)|^{2} d x \cdot E \int_{a}^{b}\left|X_{A}(x, \omega)-X(x, \omega)\right|^{2} d x
\end{array}
$$

3. A Fourier integral and an inversion formula for the Fourier transform. We shall show the analogous theorem for the process $X(x, \omega)$ to the ordinary Fourier single integral theorem.

THEOREM 3.1. If the covariance function $\rho(s, t)$ of the process $X(x, \omega)$ satisfies that

$$
\iint_{-\infty}^{\infty} \frac{|\rho(s, t)|}{(1+|s|)(1+|t|)} d s d t<\infty
$$

and has a continuous partial derivative $\partial^{2} \rho / \partial s \partial t$, then

$$
\underset{A \rightarrow \infty}{\lim .} \frac{1}{\pi} \int_{-\infty}^{\infty} X(u, \omega) \frac{\sin A(x-u)}{x-u} d u=X(x, \omega) .
$$

for every $x$.

Proof. The integral in (3.2) really exists, which is a consequence of Lemma 2.1 and the condition (3.1). Actually

$$
E\left|\int_{|u-x| \geqq B} X(u, \omega) \frac{\sin A(x-u)}{x-u} d u\right|^{2} \leqq \int_{|s| \geqq B} \int_{|t| \geqq B}\left|\rho(s+x, t+x) \frac{1}{s t}\right| d s d t
$$


which is made as small as we like, taking $B$ large enough. Also

$$
E\left|X(x, \omega) \int_{|u| \geqq B} \frac{\sin A u}{u} d u\right|^{2}=\rho(x, x)\left|\int_{|u| \geqq A B} \frac{\sin u}{u} d u\right|^{2}
$$

which is arbitrarily small. Hence the proof is reduced to showing that

$$
\underset{A \rightarrow \infty}{\lim . \operatorname{lo}} \int_{-B}^{B}[X(x+u, \omega)-X(x, \omega)] \frac{\sin A u}{u} d u=0
$$

since

$E\left|\int_{-B}^{B}[X(x+u, \omega)-X(x, \omega)] \frac{\sin A u}{u} d u\right|^{2}=\iint_{-B}^{B} \phi(u, v ; x) \frac{\sin A u}{u} \frac{\sin A v}{v} d u d v$

where

(3.4) $\phi(u, v ; x)=\Delta_{u v} \rho(x, x)=\rho(x+u, x+v)-\rho(x+u, x)-\rho(x, x+v)+\rho(x, x)$.

The standard theorem on the two-dimensional Fourier integral (Bochner [2, Chapter IX]) proves (3.3).

In the proof we have appealed to the two-dimensional Fourier integral theorem, but it is to be noted that the Dirichlet integral over a finite square was involved which is a much simpler case than the Fourier integral theorem over the whole plane.

If we impose the severe condition (1.6), then the Fourier transform $Y(y, \omega)$ is defined and because of Lemma 2.2, we have

$$
\begin{aligned}
\frac{1}{\sqrt{ }(2 \pi)} \int_{-A}^{A} Y(y, \omega) e^{i x y} d y & =\frac{1}{2 \pi} \int_{-A}^{A} e^{i x y} d y \underset{C \rightarrow-\infty, D \rightarrow \infty}{\lim _{C \rightarrow-\infty} \int_{C}^{D} X(u, \omega) e^{-i u y} d u} \\
& =\underset{c \rightarrow-\infty, D \rightarrow \infty}{\lim . \sin } \frac{1}{\pi} \int_{C}^{D} X(u, \omega) \frac{\sin A(u-x)}{u-x} d u \\
& =\frac{1}{\pi} \int_{-\infty}^{\infty} X(u, \omega) \frac{\sin A(u-x)}{u-x} d u .
\end{aligned}
$$

Hence Theorem 3.1 leads to

THEOREM 3.2. If the covariance function $\rho(s, t)$ of $X(x, \omega)$ satisfies $(1.6)$ and has a continuous partial derivative, and $Y(y, \omega)$ is its Fourier transform, then

$$
\lim _{A \rightarrow \infty} \frac{1}{\sqrt{(2 \pi)}} \int_{-A}^{A} e^{i y x} Y(y, \omega) d y=X(x, \omega)
$$

for every $x$.

This is, of course, the inversion formula for Fourier transform.

4. Summability theorems. We shall discuss the validity of the limit relation

$$
\lim _{A \rightarrow \infty} A \int_{-\infty}^{\infty} X(x+u, \omega) K(A u) d u=X(x, \omega) \int_{-\infty}^{\infty} K(u) d u .
$$


The standard theorem concerning this relation for a numerical function is well known and found in Bochner [2]. The corresponding theorem for the stationary process $X(t, \omega)$ was obtained by the author [7] and quite recently it was generalized by $\mathrm{H}$. Hatori [6].

We shall prove

THEOREM 4.1. If the covariance function $\rho(s, t)$ of the process $X(x, \omega)$ satisfies

$$
\iint_{-\infty}^{\infty} \frac{|\rho(s, t)|}{(1+|s|)(1+|t|)} d s d t \infty
$$

and the bounded function $K(x)$ satisfies that

$$
K(x)=O\left(|x|^{-1}\right) \text { as }|x| \rightarrow \infty
$$

and

$$
K(x) \in L_{1}(-\infty, \infty),
$$

then for every $x,(4.1)$ is true.

The proof will be done in the same way as for the case of a numerical function.

Proof. First of all, we have

$$
\begin{aligned}
I_{1} & =E\left|A \int_{|u|>B} X(x+u, \omega) K(A u) d u\right|^{2} \\
& =A^{2} \int_{|v|>B} \int_{|u|>B} \rho(x+u, x+v) K(A u) \overline{K(A v)} d u d v
\end{aligned}
$$

which is, in view of (4.2),

$$
\leqq C \int_{|v|>B} \int_{|u|>B} \frac{|\rho(x+u, x+v)|}{|u v|} d u d v,
$$

where $C$ is a constant. Hence for a given $\varepsilon>0$, there exists a $B$ independent of $A$ such that

$$
I_{1}<\varepsilon .
$$

Next consider, for a positive number $\delta$

$$
\begin{aligned}
I_{2} & =E\left|A \int_{B \geqq|u| \geqq \delta} X(x+u, \omega) K(A u) d u\right|^{2} \\
& =A^{2} \int_{D} \int \rho(x+u, x+v) K(A u) K \overline{(A v)} d u d v
\end{aligned}
$$


where $D$ denotes the domain $B \geqq|u| \geqq \delta, B \geqq|v| \geqq \delta$. There exists a bounded function $h(u, v)$ over $D$ such that

$$
\int_{D} \int\left|\frac{\rho(x+u, x+v)}{u v}-h(u, v)\right| d u d v<\varepsilon_{1},
$$

$\varepsilon_{1}$ being an arbitrary small positive number. We then have

$$
\begin{aligned}
I_{2} \leqq & A^{2} \int_{D} \int\left|\frac{\rho(x+u, x+v)}{u v}-h(u, v)\right||u v K(A u) K(A v)| d u d v \\
& +A^{2} \int_{D} \int|h(u, v)||u v K(A u) K(A v)| d u d v .
\end{aligned}
$$

Let us write $|u K(u)| \leqq C_{1},|h(u, v)| \leqq C_{2}$, where $C_{1}$ and $C_{2}$ are constants, $C_{2}$ being dependent on $\delta$ and $B$. The last expression does not exceed

$$
C_{1}^{2} \int_{D} \int\left|\frac{\rho(x+u, x+v)}{u v}-h(u, v)\right| d u d v+C_{2} B^{2} \iint_{A \delta}^{A B}|K(u) K(v)| d u d v
$$

which can be made as small as we like. In other words, we can find $A_{0}=A_{0}(\varepsilon, \delta, B)$ so large that if $A \geqq A_{0}$

$$
\left|I_{2}\right|<\varepsilon
$$

for an arbitrary positive $\varepsilon$.

Finally,

$$
\begin{aligned}
I_{3}= & E\left|A \int_{|u|<\delta} X(x+u, \omega) K(A u) d u-X(x, \omega) \int_{-\infty}^{\infty} K(u) d u\right|^{2} \\
= & 2 E\left|A \int_{|u|<\delta}[X(x+u, \omega)-X(x, \omega)] K(A u) d u\right|^{2} \\
& +2 E|X(x, \omega)|^{2}\left|A \int_{|u| \geqq \delta} K(A u) d u\right|^{2} \\
= & 2 A^{2} \int_{|v|<\delta} \int_{|u|<\delta} \phi(u, v) K(A u) \overline{K(A v)} d u d v+2 \rho(x, x) \int_{|u| \geqq A \delta}|K(u)| d u
\end{aligned}
$$

where $\phi(u, v)$ is $\Delta_{u v} \rho(x, x)$ as defined by (3.4).

Since $\rho(s, t)$ is continuous, we can choose $\delta$ so small that $|\phi(u, v)|<\varepsilon_{2}, \varepsilon_{2}$ being an arbitrarily small positive number. If we do this, the first term of (4.7) is less than $2 \varepsilon_{2}\left(\int_{-\infty}^{\infty}|K(u)| d u\right)^{2}$. The second term converges to zero as $A$ tends to infinity. Accordingly, we can find $\delta$ and $A_{1}$ such that for any given $\varepsilon$,

$$
I_{3}<\varepsilon
$$

if $A>A_{1}$.

If $A_{0}$ is supposed to have been determined according to $\delta$ chosen above and $A$ is taken larger than $A_{0}$ and $A_{1}$, then (4.5), (4.6) and (4.8) give 


$$
E\left|A \int_{-\infty}^{\infty} X(x+u, \omega) K(A u) d u-X(x, \omega) \int_{-\infty}^{\infty} K(u) d u\right|^{2} \leqq 3 I_{1}+3 I_{2}+3 I_{3}<9 \varepsilon,
$$

which proves the theorem.

In a similar way we may prove

THEOREM 4.2. If the covariance function $\rho(s, t)$ satisfies (4.2) and aiso

$$
\int_{-\infty}^{\infty} \frac{|\rho(s, t)|^{p}}{(1+|s|)(1+|t|)} d s d t<\infty
$$

for some $p>1$, if $K(x) \in L_{1}(-\infty, \infty)$ and

$$
\int_{-\infty}^{\infty}|K(x)|^{q}|x|^{q-1} d x<\infty
$$

where $1 / p+1 / q=1$, then (4.1) is true for every $x$.

The corresponding theorem for the ordinary function was obtained by S. Bochner and S. Izumi [3]. Theorem 4.1 is no more than the case $p=1, q=\infty$ in Theorem 4.2.

A particular case of Theorem 4.1 is

$$
\lim _{A \rightarrow \infty} \frac{1}{\pi} \int_{-\infty}^{\infty} X(u, \omega) \frac{\sin ^{2} A(x-u)}{A(x-u)^{2}} d u=X(x, \omega) .
$$

However the condition (4.2) may be relaxed to

$$
\iint_{-\infty}^{\infty} \frac{\rho(s, t)}{\left(1+|s|^{2}\right)\left(1+|t|^{2}\right)} d s d t<\infty
$$

The proof will be done in a quite similar way.

THEOREM 4.3. Under the condition (4.12), the relation (4.11) holds for every $x$. Another version of (4.10) is

THEOREM 4.4. If $\iint_{-\infty}^{\infty}|\rho(s, t)| d s d t<\infty$ and $Y(y, \omega)$ is the Fourier transform of $X(x, \omega)$, then

$$
\lim _{A \rightarrow \infty} \frac{1}{\sqrt{ }(2 \pi)} \int_{-A}^{A}\left(1-\frac{|y|}{A}\right) e^{i y x} Y(y, \omega) d y=X(x, \omega
$$

for every $x$.

5. $k$-transform. The generalized Fourier transform of a stochastic process will be defined in this section although it is well known. The one for the ordinary function was named $k$-transform by S. Bochner [2] who gave an extensive study about it. The extension to the process will be done in a similar way. In the subsequent sections we only need the case $k=2$, namely the 2-transform, but for convenience we shall give the basic definitions and properties of the $k$-transform here. 
Let us suppose that the covariance function $\rho(s, t)$ of the process $X(x, \omega)$ satisfies

$$
\iint_{-\infty}^{\infty} \frac{|\rho(s, t)|}{\left(1+|s|^{k}\right)\left(1+|t|^{k}\right)} d s d t<\infty
$$

for some positive integer $k$. In this case the integral

exists.

$$
\int_{-\infty}^{\infty} \frac{X(x, \omega)}{1+|x|^{k}} d x
$$

If $\rho(s, t)$ is bounded as it is in the stationary case, then it is obvious that (5.1) is satisfied for $k>1$.

We shall now introduce, following Bochner, the function of $x$,

$$
\begin{aligned}
L_{k}(x, \alpha) & =\sum_{j=0}^{k-1} \frac{(-i \alpha x)^{j}}{j !}, & & |x| \leqq 1, \\
& =0, & & |x|>1,
\end{aligned}
$$

and define

$$
Y_{k}(y, \omega)=\frac{1}{\sqrt{ }(2 \pi)} \int_{-\infty}^{\infty} X(x, \omega) \frac{e^{-i y x}-L_{k}(x, y)}{(i x)^{k}} d x
$$

which is easily seen to exist, if $(5.1)$ is assumed. $Y_{k}(y, \omega)$ is called the $k$-transform of $X(x, \omega)$. Also it is seen to be continuous. In fact, for an arbitrary $h$, and a positive $A$,

$\Delta_{h} Y_{k}(y, \omega)=Y_{k}(y+h, \omega)-Y_{k}(y, \omega)$

$$
\begin{aligned}
= & \frac{1}{\sqrt{(2 \pi)}} \int_{|x| \leqq 1} \frac{1}{(i x)^{k}} \cdot \sum_{j=k}^{\infty} \frac{[-i(y+h) x]^{j}-[-i y x]^{j}}{j !} \cdot X(x, \omega) d x \\
& +\frac{1}{\sqrt{ }(2 \pi)}\left\{\int_{A \geqq|x|>1}+\int_{|x|>A}\right\} \frac{e^{-i x(y+h)}-e^{-i x y}}{(-i x)^{k}} X(x, \omega) d x \\
= & \frac{1}{\sqrt{ }(2 \pi)} \int_{|x| \leqq 1} P_{1}(x, y) X(x, \omega) d x \\
& +\frac{1}{\sqrt{(2 \pi)}}\left\{\int_{A \geqq|x| \geqq 1}+\int_{|x|>A}\right\} P_{2}(x, y) X(x, \omega) d x \\
= & I_{1}+I_{2}+I_{3}
\end{aligned}
$$

say. Now we have the estimates

$$
\left|P_{1}(x, y)\right|=O(h), \quad\left|P_{2}(x, y)\right|=o(h) \quad(|x|>1)
$$

and

$$
\left|P_{2}(x, y)\right| \leqq O\left(|x|^{-k}\right) \quad(|x|>1)
$$

where $O$ and $o$ may depend on $y$ and $A$. 
We then have

$$
E\left|\Delta_{h} Y_{k}(y, \omega)\right|^{2} \leqq 3\left(E\left|I_{1}\right|^{2}+E\left|I_{2}\right|^{2}+E\left|I_{3}\right|^{2}\right)
$$

and using Lemma 2.1 and (5.5) we obtain

$$
\begin{aligned}
E\left|I_{1}\right|^{2} & =\int_{\left|x_{1}\right| \leqq 1} \int_{\left|x_{2}\right| \leqq 1} \rho\left(x_{1}, x_{2}\right) P_{1}\left(x_{1}, y\right) P_{1}\left(x_{2}, y\right) d x_{1} d x_{2} \\
& =O\left(h^{2}\right)=o(1) \text { as } h \rightarrow 0 .
\end{aligned}
$$

Similarly, $E\left|I_{2}\right|^{2}=o(1)$ if $A$ is fixed and $h$ tends to zero. Also

$$
E\left|I_{3}\right|^{2} \leqq \int_{\left|x_{1}\right| \geqq A} \int_{\left|x_{2}\right| \geqq A} \frac{\left|\rho\left(x_{1}, x_{2}\right)\right|}{\left|x_{1}\right|^{k}\left|x_{2}\right|^{k}} d x_{1} d x_{2}
$$

which is made as small as we like, if $A$ is taken large enough. These considerations prove the continuity of $Y_{k}(y, \omega)$.

If the two processes, for both of which the condition (5.1) is true with the same $k$, have the identical $k$-transform, then the processes must be identical.

This statement is proved the same way as the analogous theorem for the ordinary $k$-transform. Namely, taking the $k$ th difference of $Y_{k}(y, \omega)$

we obtain

$$
\Delta_{1}^{k} Y_{h}(y, \omega)=\sum_{j=0}^{k}(-1)^{j}\left(\begin{array}{l}
k \\
j
\end{array}\right) Y_{k}(y+k-j)
$$

$$
\Delta_{1}^{k} Y_{k}(y, \omega)=\frac{1}{\sqrt{ }(2 \pi)} \int_{-\infty}^{\infty}\left(\frac{e^{-i x}-1}{-i x}\right)^{k} X(x, \omega) e^{-i y x} d x
$$

which is the Fourier transform of

$$
X_{1}(x, \omega)=\left(\frac{e^{-i x}-1}{-i x}\right)^{k} X(x, \omega)
$$

for which the covariance function is integrable. The inversion formula (4.13) in Theorem 4.4 shows that $X_{1}(x, \omega)$ is uniquely determined which, in turn, means that $X(x, \omega)$ is determined by its $k$-transform.

Let us call the stochastic process of the form

$$
\sum_{n=0}^{k} a_{n}(\omega) y^{n}
$$

a polynomial process of degree $k$, where $a_{n}(\omega)$ is a random variable. Since the $(k+1)$ th difference of such a process is identically zero, the following statement is true.

If the k-transforms of two stochastic processes for both of which (5.1) is true, are equal to each other up to a polynomial process of degree $k-1$, then the original processes are identical. 
We now define the derivative $d Y / d y=Y^{\prime}(y, \omega)$ by

$$
\lim _{h \rightarrow 0} \frac{1}{h} \Delta_{h} Y(x, \omega)=\lim _{h \rightarrow 0} \frac{Y(y+h, \omega)-Y(y, \omega)}{h}
$$

when it exists and the successive derivatives $Y^{(k)}(y, \omega)$ by $d Y^{(k-1)} / d y, k=2,3, \cdots$.

Now if we suppose that the covariance function of $X(x, \omega)$ satisfies the condition

$$
\iint_{-\infty}^{\infty}|\rho(s, t)| d s d t<\infty
$$

then (5.1) is obviously satisfied for every positive integer $k$ so that the $k$-transform $Y_{k}(y, \omega)$ exists. In case it is seen that the $k$ th derivative of $Y_{k}(y, \omega)$ exists and

$$
Y_{k}^{(k)}(y, \omega)=Y(y, \omega)
$$

where $Y(y, \omega)$ is the Fourier transform of $X(x, \omega)$.

In order to prove this, it is sufficient to show that the derivative of $Y_{k}(y, \omega)$ is $Y_{k-1}(y, \omega)$ under the condition (5.9).

We see that

$$
\begin{aligned}
J= & \frac{1}{h} \Delta_{h} Y_{k}(y, \omega)-Y_{k-1}(y, \omega) \\
= & \frac{1}{\sqrt{ }(2 \pi)} \int_{|x| \leqq 1} P(x, y) X(x, \omega) d x \\
& \quad+\frac{1}{\sqrt{ }(2 \pi)}\left(\int_{A \geqq|x|>1}+\int_{|x|>A}\right) Q(x, y) X(x, \omega) d x \\
= & J_{1}+J_{2}+J_{3},
\end{aligned}
$$

say, where

$$
P(x, y)=\frac{1}{(-i x)^{k}} \sum_{j=k}^{\infty} \frac{(-i x)^{j}}{j !} \frac{(y+h)^{j}-y^{j}}{h}
$$

and

$$
-\frac{1}{(-i x)^{k-1}} \sum_{j=k-1}^{\infty} \frac{(-i x y)^{j}}{j}=O(h) \quad \text { for }|x| \leqq 1,
$$

$$
\begin{array}{rlrl}
Q(x, y) & =\frac{1}{(-i x)^{k}} \frac{1}{h}\left(e^{-i x(y+h)}-e^{-i x y}\right)-\frac{1}{(-i x)^{k-1}} e^{-i x y} \\
& =O(A h) & \text { for } A \geqq|x|>1 \\
& =O(1) \quad & \text { for } A<|x| .
\end{array}
$$

From these estimates we have 
and

$$
\begin{aligned}
E\left|J_{1}\right|^{2} & \leqq \frac{1}{2 \pi} \int_{|s| \leqq 1} \int_{|t| \leqq 1}|\rho(s, t)||P(s, y) P(t, y)| d s d t \\
& =O(h) \\
E\left|J_{2}\right|^{2} & \leqq \frac{1}{2 \pi} \int_{1 \leqq|s| \leqq A} \int_{1 \leqq|t| \leqq A}|\rho(s, t)||Q(s, y) Q(t, y)| d s d t \\
& \leqq O\left(A^{2} h^{2}\right)
\end{aligned}
$$

$$
\begin{aligned}
E\left|J_{3}\right|^{2} & \leqq \frac{1}{2 \pi} \int_{|s| \geqq A} \int_{|t| \geqq A}|\rho(s, t)||Q(s, y) Q(t, y)| d s d t \\
& =O\left(\int_{|s| \geqq A} \int_{|t| \geqq A}|\rho(s, t)| d s d t\right) .
\end{aligned}
$$

So $E\left|J_{3}\right|^{2}$ is arbitrarily small if $A$ is taken large enough, while $E\left|J_{1}\right|^{2}$ and $E\left|J_{2}\right|^{2}$ converge to zero as $h$ converges to zero, $A$ being kept fixed as such. Hence $E|J|^{2} \leqq 3 \Sigma_{1}^{3} E\left|J_{j}\right|^{2}$ is made arbitrarily small for sufficiently small $h$ which proves the required result.

6. Representation theorem. We shall discuss the spectral representation of a stochastic process for which the covariance function $\rho(s, t)$ satisfies the condition

$$
\iint_{-\infty}^{\infty} \frac{|\rho(s, t)|}{\left(1+s^{2}\right)\left(1+t^{2}\right)} d s d t<\infty .
$$

First of all we shall introduce the integral

$$
\int_{a}^{b} \int_{a}^{u} f(t) d^{2} Y(x, \omega)
$$

for any measurable, separable and continuous process $Y(x, \omega)$ and for any function $f(t)$ such that $f^{\prime \prime}(t)$ is bounded and continuous in $[a, b]$ and

$$
f(a)=f^{\prime}(a)=0 \text {. }
$$

The integral (6.2) is defined as

$$
f(b) Y(b, \omega)-2 \int_{a}^{b} f^{\prime}(u) Y(u, \omega) d u+\int_{a}^{b} \int_{a}^{u} f^{\prime \prime}(x) Y(x, \omega) d x d u .
$$

This type of integral is, of course, known and is obtained formally by integration by parts applied to (6.2) with $Y^{\prime \prime}(x, \omega) d x$ instead of $d^{2} Y(x, \omega)$. See, for instance, Bochner [2].

As a matter of fact, what we actually need in this section is the integral of the type

$$
\int_{0}^{T} \int_{-u}^{u} f(x) d^{2} Y(x, \omega),
$$


which is similarly defined by

where

$$
\begin{aligned}
\Delta_{T}^{2}\{f(-T) Y(-T, \omega)\}-2 \int_{0}^{T} \Delta_{2 x} f^{\prime \prime}(-x) Y(-x, \omega) d x \\
+\int_{0}^{T} \int_{-u}^{u} f^{\prime}(x) Y(x, \omega) d x d u,
\end{aligned}
$$

$$
\begin{aligned}
& \Delta_{T}^{2}\{f(-T) Y(-T, \omega)\}=f(T) Y(T, \omega)-2 f(0) Y(0, \omega)+f(-T) Y(-T, \omega), \\
& \Delta_{2 x}\left\{f^{\prime}(-x) Y(-x, \omega)\right\}=f^{\prime}(x) Y(x, \omega)-f^{\prime}(-x) Y(-x, \omega),
\end{aligned}
$$

in which we do not have to place the condition of vanishing of $f(x)$ at either of the integration limits 0 and $T$.

In particular, we have

$$
\begin{aligned}
\int_{0}^{T} \int_{-u}^{u} e^{i y x} d^{2} Y(y, \omega) d u= & \Delta_{T}^{2}\left\{e^{-i T x} Y(-T, \omega)\right\} \\
& -2 i x \int_{0}^{T} \Delta_{2 y} e^{-i y x} Y(-y, \omega) d y \\
& +(i x)^{2} \int_{0}^{T} \int_{-u}^{u} e^{i y x} Y(y, \omega) d y d u
\end{aligned}
$$

Then the following representation theorem is a simple consequence of Theorem 4.3.

THEOREM 6.1. If $X(x, \omega)$ is a stochastic process with covariance function $\rho(s, t)$ for which (6.1) is true, then it is true that

$$
\lim _{T \rightarrow \infty} \frac{1}{T} \int_{0}^{T} \int_{-u}^{u} e^{i y x} d^{2} Y_{2}(y, \omega)=X(x, \omega)
$$

for every $x$ where $Y_{2}(y, \omega)$ is the 2-transform of $X(x, \omega)$.

Proof. Write

$$
\phi(y, s)=\frac{e^{-i s y}-L_{2}(y, s)}{(-i s)^{2}}
$$

The 2-transform becomes

$$
Y_{2}(y, \omega)=\frac{1}{\sqrt{(2 \pi)}} \int_{-\infty}^{\infty} X(s, \omega) \phi(y, s) d s .
$$

Inserting this into $(6.6)$ in place of $Y(y, \omega)$ yields that the integral on the lefthand side of (6.7) is

$$
\frac{1}{2 \pi} \frac{1}{T} \int_{-\infty}^{\infty} K(T, s ; x) X(s, \omega) d s
$$

where 


$$
\begin{aligned}
K(T, s ; x)= & e^{i T x} \phi(T, s)+e^{-i T x} \phi(-T, s)-2 \phi(0, s)-2 i x \int_{0}^{T} \phi(y, s) e^{i y x} d y \\
& +2 i x \int_{0}^{T} \phi(-y, s) e^{-i y x} d y-x^{2} \int_{0}^{T} \int_{-u}^{u} \phi(y, s) d y d u \\
= & \int_{0}^{T} \int_{-u}^{u} e^{i y x} \frac{\partial^{2}}{\partial y^{2}} \phi(y, s) d y d u \\
= & \frac{4 \sin ^{2}(T(s-x) / 2)}{(s-x)^{2}} .
\end{aligned}
$$

Hence (6.9) turns out to be

$$
\frac{1}{\pi} \int_{-\infty}^{\infty} \frac{\sin ^{2}(T(s-x) / 2)}{(s-x)^{2} T / 2} X(s, \omega) d s,
$$

which, in view of Theorem 4.3 converges to $X(x, \omega)$ for every $x$.

7. Harmonizable processes. There is another case where some representation theorem is available; that is, the case where the corresponding covariance function admits the representation

$$
\rho(s, t)=\int_{-\infty}^{\infty} \int^{i \xi s-i \eta t} d_{2} F(\xi, \eta)
$$

where $F(\xi, \eta)$ is a function of bounded variation over $\boldsymbol{R}_{2}$. Such a process was named by $M$. Loève [8], [9] a harmonizable process and basic properties concerning it have been investigated by him.

For the sake of completeness we shall pick up here one of his results in connection with the generalized transform.

Although the covariance function $\rho(s, t)$ of a given process $X(x, \omega)$ for which (7.1) is true does not necessarily satisfy the condition (5.1) with $k=1$, still the following symmetric 1-transform

$$
\lim _{T \rightarrow \infty} \frac{1}{\sqrt{(2 \pi)}} \int_{-T}^{T} \frac{e^{-i y x}-L_{1}(y, x)}{-i x} X(x, \alpha) d x=Y_{1}^{*}(y, \omega)
$$

is proved to exist for every $y$. For, after some familiar manipulation, we see, writing

that

$$
\begin{aligned}
I_{T, T^{\prime}} & =\int_{T \leqq|x| \leqq T^{\prime}} \frac{e^{-i y x}-L_{1}(y, x)}{-i x} X(x, \omega) d x \\
& =\int_{T \leqq|x| \leqq T^{\prime}} \frac{e^{-i y x}}{-i x} X(x, \omega) d x, \quad T^{\prime}>T>1,
\end{aligned}
$$




$$
E\left|I_{T, T^{\prime}}\right|^{2}=4 \int_{-\infty}^{\infty} \int_{-\infty}^{T^{\prime}} d F(\xi, \eta) \int_{T} \frac{\sin s(y-\xi) \sin t(y-\eta)}{s t} d s d t
$$

which converges to zero as $T$ and $T^{\prime}$ tend to infinity.

Now we define the integral

$$
\int_{-T}^{T} e^{i y x} d Y_{1}^{*}(y, \omega)=e^{i T x} Y_{1}^{*}(T, \omega)-e^{-i T x} Y_{1}^{*}(-T)-i x \int_{-T}^{T} Y_{1}^{*}(y) e^{i y x} d y
$$

Then we have

THEOREM 7.1. For every $x$

$$
\lim _{T \rightarrow \infty} \frac{1}{\sqrt{(2 \pi)}} \int_{-T}^{T} e^{i y x} d Y_{1}^{*}(y, \omega)=X(x, \omega) .
$$

This was obtained by $M$. Loève. The proof will be outlined here using our definition of the integral. Using Lemma 2.2 in order to exchange the order of integration and noting that

$$
\begin{aligned}
e^{i T x} \phi(T, s)-e^{-i T x} \phi(-T, s)-i x \int_{-T}^{T} e^{i y x} \phi(y, s) d y & =\int_{-T}^{T} e^{i y x} \frac{\partial}{\partial y} \phi(y, s) d y \\
& =2 i \frac{\sin T(x-s)}{x-s},
\end{aligned}
$$

where $\phi(y, s)=\left(e^{-i y s}-L_{1}(y, s)\right) /(-i s)$, we see that

$$
J=\frac{1}{\sqrt{ }(2 \pi)} \int_{-T}^{T} e^{i y x} d Y_{1}^{*}(y, \omega)=\frac{1}{\pi} \int_{-\infty}^{\infty} \frac{\sin T(x-s)}{x-s} X(s, \omega) d s,
$$

and also

$$
\begin{aligned}
& E|J-X(x, \omega)|^{2}=E \mid \text { 1.i.m. }\left.\frac{1}{\pi} \int_{-A}^{A} \frac{\sin T(x-s)}{x-s}[X(s, \omega)-X(x, \omega)] d s\right|^{2} \\
& =\lim _{\Lambda \rightarrow \infty} \int_{-A}^{A} \int_{-A}[\rho(s, t)-\rho(s, x)-\rho(x, t)+\rho(x, x)] \frac{\sin T(x-s) \sin T(x-t)}{x-s} d s d t \\
& =\frac{4}{\pi^{2}} \int_{-\infty}^{\infty} \int_{2} F(\xi, \eta) e^{i x(\xi+\eta) / 2} \\
& \cdot \lim _{A \rightarrow \infty} \int_{-A}^{A} e^{i s \xi / 2} \frac{\sin (s-x) \xi}{2} \frac{\sin T(s-x)}{s-x} d s \\
& \cdot \lim _{A \rightarrow \infty} \int_{-A}^{A} e^{i t \eta / 2} \frac{\sin (t-x) \eta}{2} \frac{\sin T(t-x)}{t-x} d t \\
& =0 \text {, }
\end{aligned}
$$

because 


$$
\begin{array}{rlr}
\lim _{A \rightarrow \infty} \int_{-A}^{A} e^{i s \xi / 2} \frac{\sin (s-x) \xi}{2} \frac{\sin T(s-x)}{s-x} d s & \\
=e^{i \xi x} \int_{-\infty}^{\infty}\left(e^{i \lambda} \sin \lambda \sin (s T / \xi) / \lambda\right) d \lambda, & \xi \neq 0, \\
=0, & \xi=0,
\end{array}
$$

which converges to 0 boundedly if $T$ goes to infinity and the same thing is true for the other integral in (7.5).

8. Covariances of $Y_{2}(y, \omega)$ and $Y_{1}^{*}(y, \omega)$. We have shown that any nonstationary process, under a very general condition, admits the spectral representation in terms of $Y_{2}(y, \omega)$. We shall, in this section, find the covariance function of $Y_{2}(y, \omega)$ and also of $Y_{1}^{*}(y, \omega)$, the representation of a harmonizable process.

As we did before, we suppose that

$$
\iint_{-\infty}^{\infty} \frac{|\rho(s, t)|}{\left(1+s^{2}\right)\left(1+t^{2}\right) !} d s d t<\infty .
$$

It will be convenient to think of the covariance of the difference of $Y_{2}(y, \omega)$ of the second order

$$
\Delta_{h}^{2} Y_{2}(y-h, \omega)=Y_{2}(y+h, \omega)-2 Y_{2}(y, \omega)+Y_{2}(y-h, \omega)
$$

which is immediately seen to be

$$
\frac{1}{\sqrt{ }(2 \pi)} \int_{-\infty}^{\infty} X(x, \omega) \frac{\sin ^{2}(h / 2) x}{(x / 2)^{2}} e^{-i y x} d x
$$

Writing

we find

$$
E \Delta_{h}^{2} Y_{2}(u-h, \omega) \overline{\Delta_{k}^{2} Y_{2}(v-k, \omega)}=\rho\left(u, v ; h, k ; \Delta^{2} Y_{2}\right),
$$

(8.3) $\rho\left(u, v ; h, k ; \Delta^{2} Y_{2}\right)=$

$$
\frac{1}{2 \pi} \int_{-\infty}^{\infty} \rho(x, y) \frac{\sin ^{2} h(x / 2)}{(x / 2)^{2}} \frac{\sin ^{2} k(y / 2)}{(y / 2)^{2}} e^{-i u x+i v y} d x d y
$$

and in particular the variance $\rho\left(u, u ; h, h ; \Delta^{2} Y_{2}\right)$ of $\Delta_{h}^{2} Y_{2}(y-h, \omega)$ is

$$
\frac{1}{2 \pi} \iint_{-\infty}^{\infty} \rho(x, y) \frac{\sin ^{2} h(x / 2)}{(x / 2)^{2}} \frac{\sin ^{2} h(y / 2)}{(y / 2)^{2}} e^{-i u(x-y)} d x d y .
$$

Next we shall compute the covariance function

$$
\rho\left(u, v ; h, k ; \Delta Y_{1}^{*}\right)=E \Delta_{h} Y_{1}^{*}(u-h / 2, \omega) \overline{\Delta_{k} Y_{1}^{*}(v-k / 2, \omega)}
$$




$$
\Delta_{h} Y_{1}^{*}(y, \omega)=Y_{1}^{*}(y+h / 2, \omega)-Y_{1}^{*}(y-h / 2, \omega),
$$

where $Y_{1}^{*}$ is the process in the harmonic representation of a harmonizable process, given in the preceding section. It is immediate that

$$
\begin{aligned}
\rho\left(u, v ; h, k ; \Delta_{h} Y_{1}\right)= & \frac{1}{2 \pi} \iint_{-\infty}^{\infty} \rho(x, y) \frac{\sin h(x / 2)}{(x / 2)} \frac{\sin k(y / 2)}{(y / 2)} e^{-i u x+i v y} d x d y \\
= & \frac{1}{2 \pi} \int_{-\infty}^{\infty} \int_{-\infty} d F(\xi, \eta) \int_{-\infty}^{\infty} e^{i(\xi-u) x} \frac{\sin h(x / 2)}{x / 2} d x \\
& \cdot \int_{-\infty}^{\infty} e^{-i(\eta-v) y} \frac{\sin k(y / 2)}{(y / 2)} d y \\
= & 2 \pi \iint_{-\infty}^{\infty} D_{h / 2}(\xi-u) D_{k} / 2(\eta-v) d F(\xi, \eta),
\end{aligned}
$$

where

$$
\begin{aligned}
D_{\lambda}(z) & =1, & & |z|<\lambda, \\
& =\frac{1}{2}, & & |z|=\lambda, \\
& =0, & & |z|>\lambda .
\end{aligned}
$$

Hence we finally have

$$
\begin{aligned}
\rho\left(u, v ; h, k ; \Delta Y_{1}^{*}\right)= & 2 \pi[F(u+h / 2, v+k / 2)-F(u+h / 2, v-k / 2) \\
& -F(u-h / 2, v+k / 2)+F(u-h / 2, v-k / 2)]
\end{aligned}
$$

if the four points $(u \pm h / 2, v \pm k / 2)$ are continuity points of $F(\xi, \eta)$.

The variance $\rho\left(u, u ; h, h ; \Delta Y_{1}^{*}\right)$ is the difference of $F(\xi, \eta)$ in the square $|\xi-u| \leqq h / 2,|\eta-u| \leqq h / 2$.

From this it is easily seen from (8.6) that if the difference

$$
\Delta_{2} F(\xi, \eta)=F\left(\xi_{2}, \eta_{2}\right)-F\left(\xi_{2}, \eta_{1}\right)-F\left(\xi_{1}, \eta_{2}\right)+F\left(\eta_{1}, \eta_{2}\right)
$$

over the rectangle $\xi_{1} \leqq \xi \leqq \xi_{2}, \eta_{1} \leqq \eta \leqq \eta_{2}$ which is completely contained in the $\xi, \eta$ domain

$$
\left|\frac{\xi}{h}-\frac{\eta}{k}\right|>1,
$$

is zero, then the covariance function $\rho\left(u, v ; h, k ; \Delta Y^{*}\right)$ is zero in the domain in the $u, v$ plane

$$
\left|\frac{u}{h}-\frac{v}{k}\right|>2 \text {. }
$$


If the process $X(x, \omega)$ is stationary in the wide sense, namely, the corresponding $\rho(s, t)$ is a function only of the difference $s-t$, then, as is well known, it admits the harmonic representation

$$
\int_{-\infty}^{\infty} e^{i(s-t) \xi} d F(\xi)
$$

where $F(\xi)$ is a bounded nondecreasing function and so is the harmonizable process where the whole mass of the corresponding $F(\xi, \eta)$ is concentrated on the line $\xi=\eta$. So the variation of $F(\xi, \eta)$ is zero over every closed rectangle in the domain $|\xi-\eta|>0$, because in (8.7) $h=k$ can be taken arbitrarily small. Hence the covariance function $\rho\left(u, v ; h, k ; \Delta Y_{1}^{*}\right)$ vanishes if $(u-h / 2, u+h / 2)$ and $(v-k / 2, v+k / 2)$ have no common point. This is, of course, a basic property of the stationary process.

9. A two-dimensional Wiener formula. In the subsequent section, we shall deal with the limit value of

$$
\frac{1}{2 T} \int_{-T}^{T} X(x, \omega) d x
$$

In doing this, we shall need the following two-dimensional Wiener formula in Theorem 9.1. It will also be of some interest of its own.

THEOREM 9.1. Suppose that $K(x, y)$ is a function such that

$$
\iint_{0}^{\infty}|K(x, y)| d x d y<\infty,
$$

$\partial^{2} K(x, y) / \partial x \partial y$ exists and is integrable over every finite interval and there exists a constant $C$ such that

$$
\left(1+x^{2}\right)\left(11+y^{2}\right)|K(x, y)| \leqq C \quad \text { for } 0 \leqq x, y<\infty .
$$

If a given function $f(x, y)$ satisfies the condition that

$$
\lim _{T, T^{\prime} \rightarrow \infty} \frac{1}{T T^{\prime}} \int_{0}^{T^{\prime}} \int_{0}^{T} f(x, y) d x d t=m
$$

exists no matter how $T$ and $T^{\prime}$ tend to infinity and

$$
\frac{1}{T T^{\prime}} \int_{0}^{T^{\prime}} \int_{0}^{T}|f(x, y)| d x d y \leqq D
$$

for any $T, T^{\prime}>0$, where $D$ is a constant, then 


$$
\lim _{\varepsilon \rightarrow 0} \int_{0}^{\infty} \int_{0} f\left(\frac{x}{\varepsilon} \frac{y}{\varepsilon}\right) K(x, y) d x d y=m \int_{0}^{\infty} \int_{0} K(x, y) d x d y .
$$

It will be convenient to state the following lemma.

LEMMA 9.1. If (9.3) is satisfied for any $T, T^{\prime}>0$, then

$$
\int_{A^{\prime}}^{B^{\prime}} \int_{A}^{B} \frac{|f(x, y)|}{\left(1+\alpha^{2} x^{2}\right)\left(1+\alpha^{2} y^{2}\right)} d x d y \leqq \frac{36 D}{\alpha^{2}(1+\alpha A)\left(1+\alpha A^{\prime}\right)}
$$

where $\alpha$ is any positive constant and $A, A^{\prime}>0$.

Proof. Write

$$
\begin{aligned}
& \int^{u}|f(x, y)| d x=\Phi(u, y), \\
& \int_{0}^{v}|| f(x, y) \mid d x d y=\Psi(u, v) .
\end{aligned}
$$

Now a repeated applicatio o ntegration by parts shows for $0 \leqq A<B$ $0 \leqq A^{\prime}<B^{\prime}$

$$
\begin{aligned}
& \int_{A^{\prime}}^{B^{\prime}} \int_{A}^{B} \frac{|f(x, y)|}{\left(1+\alpha^{2} x^{2}\right)\left(1+\alpha^{2} y^{2}\right)} d x d y \\
& \leqq \frac{1}{1+\alpha^{2} B^{2}} \int_{A^{\prime}}^{B^{\prime}} \frac{\Phi(B, y)}{1+\alpha^{2} y^{2}} d y+2 \alpha^{2} \int_{A^{\prime}}^{B^{\prime}} \frac{d y}{1+\alpha^{2} y^{2}} \int_{A}^{B} \frac{x \Phi(x, y)}{\left(1+\alpha^{2} x^{2}\right)^{2}} d x \\
& \leqq \frac{\Psi\left(B, B^{\prime}\right)}{\left(1+\alpha^{2} B^{2}\right)\left(1+\alpha^{2} B^{2}\right)}+\frac{2 \alpha^{2}}{1+\alpha^{2} B^{2}} \int_{A^{\prime}}^{B^{\prime}} \frac{y \Psi(B, y)}{\left(1+\alpha^{2} y^{2}\right)^{2}} d y \\
&+2 \alpha^{2} \int_{A}^{B} \frac{x}{\left(1+\alpha^{2} x^{2}\right)^{2}} d x \int_{A^{\prime}}^{B^{\prime}} \frac{\Phi(x, y)}{1+\alpha^{2} y^{2}} d y \\
& \leqq \frac{2 \alpha^{2}}{\left(1+\alpha^{2} B^{2}\right)\left(1+B^{\prime} B^{\prime 2}\right)}+\frac{2 \alpha^{2} B^{2}}{1+B^{\prime}} \frac{y \Psi(B, y)}{\left(1+\alpha^{2} y^{2}\right)^{2}} d y \\
&+\frac{2 \alpha^{2}}{1+\alpha^{2} B^{\prime 2}} \int_{A}^{B} \frac{x \Psi\left(x, B^{\prime}\right)}{\left(1+\alpha^{2} x^{2}\right)^{2}} d x \\
&+4 \alpha^{4} \int_{A}^{B} \frac{x}{\left(1+\alpha^{2} x^{2}\right)^{2}} d x \int_{A^{\prime}}^{B^{\prime}} \frac{y \Psi(x, y)}{\left(1+\alpha^{2} y^{2}\right)^{2}} d y
\end{aligned}
$$

Because of (9.4), the last expression does not exceed 


$$
\begin{gathered}
\frac{D B B^{\prime}}{\left(1+\alpha^{2} B^{2}\right)\left(1+\alpha^{2} B^{\prime 2}\right)}+\frac{2 \alpha^{2} B}{1+\alpha^{2} B^{2}} \int_{A^{\prime}}^{B^{\prime}} \frac{y^{2}}{\left(1+\alpha^{2} y^{2}\right)^{2}} d y \\
+\frac{2 D B^{\prime} \alpha^{2}}{1+\alpha^{2} B^{\prime 2}} \int_{A}^{B} \frac{x^{2}}{\left(1+\alpha^{2} x^{2}\right)} d x \\
+4 D \alpha^{4} \int_{A}^{B} \frac{x^{2}}{\left(1+\alpha^{2} x^{2}\right)^{2}} d x \int_{A^{\prime}}^{B^{\prime}} \frac{y^{2}}{\left(1+\alpha^{2} y^{2}\right)^{2}} d y \\
\leqq \frac{D B B^{\prime}}{\left(1+\alpha^{2} B^{2}\right)\left(1+\alpha^{2} B^{\prime 2}\right)}+\frac{4 B}{\left(1+\alpha^{2} B^{2}\right)\left(1+\alpha^{2} A\right)} \\
+\frac{4 D B^{\prime}}{\left(1+\alpha^{2} B^{\prime 2}\right)(1+\alpha A)}+\frac{16 D}{\alpha^{2}(1+\alpha A)\left(1+\alpha A^{\prime}\right)} \\
\leqq \frac{36 D}{\alpha^{2}(1+\alpha A)\left(1+\alpha A^{\prime}\right)}
\end{gathered}
$$

which proves the lemma.

Proof of Theorem 9.1. It is mentioned that the integral on the left side of (9.5) exists, because of the lemma. Now we shall prove (9.5). We may suppose that $m=0$ without loss of generality and we are going to prove

$$
\lim _{\varepsilon \rightarrow 0} \int_{0}^{\infty} \int_{0} f\left(\frac{x}{\varepsilon}, \frac{y}{\varepsilon}\right) K(x, y) d x d y=0 .
$$

Now we split this integral into several parts

$$
\begin{aligned}
I & =\int_{0}^{a} \int_{0}^{a}+\int_{0}^{a} \int_{a}^{A}+\int_{a}^{A} \int_{0}^{a}+\int_{a}^{A} \int_{a}^{A}+\int_{0}^{A} \int_{A}^{\infty}+\int_{A}^{\infty} \int_{0}^{A}+\int_{A}^{\infty} \int_{A}^{\infty} \\
& =I_{1}+I_{2}+I_{3}+I_{4}+I_{5}+I_{6}+I_{7}
\end{aligned}
$$

say, where $A>1>a>0$.

First of all, we have, because of (9.3), that

$$
\begin{aligned}
\left|I_{7}\right| & \leqq \iint_{A}^{\infty}\left|f\left(\frac{x}{\varepsilon}, \frac{y}{\varepsilon}\right) K(x, y)\right| d x d y \\
& \leqq C \varepsilon^{2} \iint_{\Lambda / \varepsilon}^{\infty} \frac{|f(u, v)|}{\left(1+\varepsilon^{2} u^{2}\right)\left(1+\varepsilon^{2} v^{2}\right)} d u d v .
\end{aligned}
$$

Using Lemma 9.1, we see that

$$
\left|I_{7}\right| \leqq \frac{36 C D}{(1+A)^{2}}
$$


For a given small number $\delta>0$, we can find $A$ so that

$$
\left|I_{7}\right|<\delta \text {. }
$$

In a similar way

$$
\left|I_{6}\right| \leqq C \varepsilon^{2} \int_{A / \varepsilon}^{\infty} \int_{0}^{A / \varepsilon} \frac{|f(u, v)|}{\left(1+\varepsilon^{2} u^{2}\right)\left(1+\varepsilon^{2} v^{2}\right)} d u d v \leqq \frac{36 C D}{1+A} .
$$

$A$ can be taken such that

$$
\left|I_{6}\right|<\delta
$$

is also true.

In the same way, we also have

$$
\left|I_{5}\right| \leqq \frac{36 C D}{1+A}<\delta .
$$

In handling the remaining integrals, we shall introduce the functions

$$
F_{\varepsilon}(u, y)=\int_{0}^{u} f\left(\frac{x}{\varepsilon}, \frac{y}{\varepsilon}\right) d x,
$$

and

$$
G_{\varepsilon}(u, v)=\int_{0}^{v} F_{\varepsilon}(u, v) d v .
$$

Now the repeated application of integration by parts shows that

$$
\begin{aligned}
I_{3}= & \int_{a}^{A} \int_{0}^{a} f\left(\frac{x}{\varepsilon}, \frac{y}{\varepsilon}\right) K(x, y) d x d y \\
= & \int_{a}^{A} F_{\varepsilon}(a, y) K(a, y) d y-\int_{a}^{A} \int_{0}^{a} F(x, y) \frac{\partial}{\partial x} K(x, y) d x d y \\
(9.13)= & G_{\varepsilon}(a, A) K(a, A)-G_{\varepsilon}(a, a) K(a, a)-\int_{0}^{a} \int_{a}^{A} F(x, y) \frac{\partial}{\partial x} K(x, y) d y d x \\
= & G_{\varepsilon}(a, A) K(a, A)-G_{\varepsilon}(a, a) K(a, a)-\left.\int_{0}^{a} G_{\varepsilon}(x, A) \frac{\partial}{\partial x} K(x, y)\right|_{y=A} d x \\
& +\left.\int_{0}^{a} G_{\varepsilon}(x, a) \frac{\partial}{\partial x} K(x, y)\right|_{y=A} d x+\int_{0}^{a} \int_{a}^{A} G_{\varepsilon}(x, y) \frac{\partial^{2}}{\partial x \partial y} K(x, y) d y d x .
\end{aligned}
$$

Now we recall that, owing to $(9.5)$,

$$
G_{\varepsilon}|(u, v)| \leqq D u v
$$


for every pair of $u$ and $v$ while $D$ is a constant independent of $\varepsilon$ and also, or a given arbitrarily small $\delta>0$ there exists $\varepsilon_{0}$ such that if $\varepsilon \leqq \varepsilon_{0}$,

$$
\left|G_{\varepsilon}(u, v)\right| \leqq u v
$$

provided $u>a>0, v>a>0, \varepsilon_{0}$ being independent of $u$ and $v$, but dependent on $a$.

Since, in view of (9.3), (9.14),

$$
\begin{array}{ll}
\left|G_{\varepsilon}(a, A) K(a, A)\right| & \leqq D a A \frac{C}{\left(1+a^{2}\right)\left(1+A^{2}\right)} \leqq \frac{C D a}{A}, \\
\left|G_{\varepsilon}(a, a) K(a, a)\right| & \leqq C D a^{2}, \\
\left|\int_{0}^{a} G_{\varepsilon}(x, A) \frac{\partial}{\partial x} K(x, A) d x\right| \leqq D A \int_{0}^{a} x\left|\frac{\partial}{\partial x} K(x, A)\right| d x & \leqq \int_{0}^{a} G_{\varepsilon}(x, a) \frac{\partial}{\partial x} K(x, a) d x\left|\leqq D a \int_{0}^{a} x\right| \frac{\partial}{\partial x} K(x, a) \mid d x
\end{array}
$$

and

$$
\left|\int_{0}^{a} \int_{a}^{A} G_{\varepsilon}(x, y) \frac{\partial^{2}}{\partial x \partial y} K(x, y) d y d x\right| \leqq D \int_{0}^{a} x d x \int_{0}^{A} y\left|\frac{\partial^{2}}{\partial x \partial y} K(x, y)\right| d y,
$$

the right side of (9.16) is made to be less than $\delta$ if $A$ is taken large enough and then $a$ is taken so small that (9.17)-(9.20) are very small.

Hence we have

$$
\left|I_{3}\right|<\delta
$$

Likewise, we have

$$
\left|I_{2}\right|<\delta
$$

It is easy to get

$$
\left|I_{1}\right|<\delta
$$

if $a$ is small enough.

Finally we shall deal with $I_{4}$. Now integration by parts again shows

$$
\begin{aligned}
I_{4}=G_{\varepsilon}(A, A) & K(A, A)-G_{\varepsilon}(A, a) K(A, a)-G_{\varepsilon}(a, A) K(a, A)+G_{\varepsilon}(a, a) K(a, a) \\
& -\int_{a}^{A} G_{\varepsilon}(A, y) \frac{\partial}{\partial y} K(A, y) d y+\int_{a}^{A} G_{\varepsilon}(a, y) \frac{\partial}{\partial y} K(a, y) d y \\
& -\int_{a}^{A} G_{\varepsilon}(x, A) \frac{\partial}{\partial x} K(x, A) d x+\int_{a}^{A} G_{\varepsilon}(x, a) \frac{\partial}{\partial x} K(x, a) d x \\
& +\int_{a}^{A} \int_{a}^{A} G_{\varepsilon}(x, y) \frac{\partial^{2}}{\partial x \partial y} K(x, y) d x d y .
\end{aligned}
$$


Now $A$ and $a$ are taken so that all of (9.10), (9.11), (9.12), (9.21), (9.22) and (9.23) are true. Then we let $\varepsilon$ tend to zero. Since all the terms of $I_{4}$ above involve $G_{\varepsilon}$, we see that, because of $(9.15)$,

$$
\limsup _{\varepsilon \rightarrow 0}\left|I_{4}\right|=0 .
$$

Now, we have

$$
\limsup _{\varepsilon \rightarrow 0}|I| \leqq 6 \delta,
$$

which proves the theorem.

10. Wiener formula for stochastic processes and a law of large numbers. We shall deal with the limit value of

$$
\underset{T \rightarrow \infty}{\operatorname{li.m.}} \frac{1}{T} \int_{0}^{T} X(x, \omega) d x .
$$

The existence of this limit is assured if it is assumed for the covariance function $\rho(s, t)$ of $X(x, \omega)$ that

$$
\lim _{S \rightarrow \infty, T \rightarrow \infty} \frac{1}{S T} \int_{0}^{S} \int_{0}^{T} \rho(s, t) d t d s
$$

exists and has the same value no matter how $S$ and $T$ tend to infinity.

We agree that (10.2) implies, for instance, that

exists for every $S$ and

$$
\lim _{T \rightarrow \infty} \frac{1}{T} \int_{0}^{s} \int_{0}^{T} \rho(s, t) d t d s
$$

$$
\lim _{s \rightarrow \infty} \lim _{T \rightarrow \infty} \frac{1}{S T} \int_{0}^{s} \int_{0}^{T} \rho(s, t) d t d s
$$

exists. The same holds if the order of the limits is interchanged. Notice that the limit value (10.2) is always real. The above fact follows immediately, since, writing

$$
M_{T}(\omega)=\frac{1}{T} \int_{0}^{T} X(x, \omega) d x,
$$

$$
\begin{aligned}
E\left|M_{T}(\omega)-M_{T^{\prime}}(\omega)\right|^{2}= & \frac{1}{T^{2}} \iint_{0}^{T} \rho(x, y) d x d y \\
& +\frac{1}{T^{\prime 2}} \iint_{0}^{T^{\prime}} \rho(x, y) d x d y-\frac{2}{T T^{\prime}} R \int_{0}^{T} \int_{0}^{T^{\prime}} \rho(x, y) d x d y
\end{aligned}
$$

which converges to zero as $T \rightarrow \infty, T^{\prime} \rightarrow \infty$ in an arbitrary manner. 
We denote

$$
\underset{T \rightarrow \infty}{\lim .} M_{T}(\omega)=M(\omega) \text {. }
$$

We shall now prove

THEOREM 10.1. If (10.2) exists no matter how $S$ and $T$ may tend to infinity and

$$
\int_{0}^{T} \int_{0}^{S}|\rho(x, y)| d x d y \leqq D S T
$$

for every $S, T>0, D$ being a constant and if, moreover, $K(x)$ is a differentiable function such that

$$
\left(1+x^{2}\right)|K(x)| \leqq C \quad \text { for } 0 \leqq x<\infty,
$$

C being a constant,

$$
\int_{0}^{\infty}|K(x)| d x<\infty
$$

and $K^{\prime}(x)$ is integrable over every finite interval, then

$$
\underset{\varepsilon \rightarrow 0}{\lim . \mathrm{f}} \int_{0}^{\infty} X\left(\frac{x}{\varepsilon}, \omega\right) K(x) d x=M(\omega) \int_{0}^{\infty} K(x) d x .
$$

Proof. First of all we shall notice that the integral on the left side of (10.8) really exists for every $\varepsilon>0$. In fact

$$
\begin{aligned}
E\left|\int_{A}^{B} X\left(\frac{x}{\varepsilon}, \omega\right) K(x) d x\right|^{2} & =\iint_{A}^{B} \rho\left(\frac{x}{\varepsilon}, \frac{y}{\varepsilon}\right) K(x) \overline{K(y)} d x d y \\
& \leqq C \iint_{A}^{B} \rho\left(\frac{x}{\varepsilon}, \frac{y}{\varepsilon}\right) \frac{d x d y}{x^{2} y^{2}}=O\left(\frac{1}{A^{2}}\right)
\end{aligned}
$$

owing to (10.6) and Lemma 9.1.

Next we shall show, writing

that

$$
f(x, y)=E[X(x, \omega)-M(\omega)] \overline{[X(y, \omega)}-\overline{M(\omega)}]
$$

and

$$
\lim _{S \rightarrow \infty, T \rightarrow \infty} \frac{1}{S T} \int_{0}^{T} \int_{0}^{S} f(x, y) d x d y=0
$$

where $D_{1}$ is some constant independent of $S$ and $T$.

(10.9) immediately follows from

$$
\frac{1}{S T} \int_{0}^{T} \int_{0}^{S}|f(x, y)| d x d y \leqq D_{1}
$$




$$
\lim _{s \rightarrow \infty} \frac{1}{s} \int_{0}^{s} E X(x, \omega) \overline{M(\omega)} d x=m,
$$

where

$$
\lim _{S \rightarrow \infty, T \rightarrow \infty} \frac{1}{S T} \int_{0}^{T} \int_{0}^{S} \rho(x, y) d x d y=m .
$$

(10.11) will be verified in the following way.

$$
\begin{aligned}
E|M(\omega)|^{2} & =E M(\omega) \overline{M(\omega)} \\
& =\lim _{S \rightarrow \infty} \lim _{T \rightarrow \infty} E\left(\frac{1}{S} \int_{0}^{S} X(x, \omega) d x \cdot \frac{1}{T} \int_{0}^{T} \overline{X(x, \omega)} d y\right) \\
& =\lim _{S \rightarrow \infty} \lim _{T \rightarrow \infty} \frac{1}{S T} \int_{0}^{T} \int_{0}^{S} \rho(x, y) d x d y=m .
\end{aligned}
$$

(10.12) is also very easy to prove.

The proof of (10.10) is not difficult either, (10.5) being used.

With these preparations, the proof of (10.8) is now immediate, by Theorem 9.1 because

$$
\begin{aligned}
E \mid \int_{0}^{\infty} X\left(\frac{x}{\varepsilon}, \omega\right) & K(x) d x-\left.M(\omega) \int_{0}^{\infty} K(x) d x\right|^{2} \\
& =\iint_{0}^{\infty} f\left(\frac{x}{\varepsilon}, \frac{y}{\varepsilon}\right) K(x) K(y) d x d y .
\end{aligned}
$$

$K(x, y)=K(x) K(y)$ satisfies the condition in Theorem 9.1, and hence (10.13) converges to zero because of (10.9) as $\varepsilon \rightarrow 0$.

Considering $X(-x, \omega)$ instead of $X(x, \omega)$ we have the following:

THEOREM 10.2. Let us suppose that

$$
\frac{1}{T S} \int_{0}^{T} \int_{0}^{S} \rho(x, y) d x d y
$$

converges as $S \rightarrow \infty, T \rightarrow \infty$ and also as $S \rightarrow-\infty, T \rightarrow \infty$ in an arbitrary manner and

$$
\frac{1}{T S} \int_{0}^{T} \int_{0}^{S}|\rho(x, y)| d x d y \leqq D
$$

for $S>0, T>0$ and for $S<0, T<0$ as well. Also, suppose that $K(x)$ is a differentiable function of $L_{1}(-\infty, \infty), x K^{\prime}(x)$ is integrable over every finite interval and satisfies

$$
\left(1+x^{2}\right)|K(x)| \leqq C, \quad-\infty<x<\infty,
$$

$C$ being a constant. Then we have 
$(10.14)$

$$
\lim _{\varepsilon \rightarrow 0} \int_{-\infty}^{\infty} X\left(\frac{x}{\varepsilon}, \omega\right) K(x) d x=M_{1}(\omega) \int_{-\infty}^{\infty} K(x) d x,
$$

where

$$
M_{1}(\omega)=\lim _{T \rightarrow \infty} \frac{1}{2 T} \int_{-T}^{T} X(x, \omega) d x
$$

Notice that the limit in (10.15) exists under the conditions of the theorem. We also mention that the definition of $M_{1}(\omega)$ in (10.15) may be replaced by

$$
M_{1}(\omega)=\lim _{T \rightarrow \infty} \frac{1}{2 T} \int_{0}^{T} X(x, \omega) d x+\lim _{T^{\prime} \rightarrow \infty} \frac{1}{2 T^{\prime}} \int_{-T^{\prime}}^{0} X(x, \omega) d x .
$$

As a particular case of this theorem, we have, under the conditions on $\rho(x, y)$,

$$
\lim _{\varepsilon \rightarrow 0} \frac{1}{\pi} \int_{-\infty}^{\infty} X(x, \omega) \frac{\sin ^{2} \varepsilon x}{x^{2}} d x=M_{1}(\omega)
$$

Now let us suppose

$$
\int_{0}^{T} \int_{0}^{S}|\rho(x, y)| d x d y \leqq D|S T|
$$

for every $-\infty<S, T<\infty$. It is then easy to see from Lemma 9.1 that

$$
\int_{-\infty}^{\infty} \frac{|\rho(x, y)|}{\left(1+x^{2}\right)\left(x+y^{2}\right)} d x d y<\infty \text {. }
$$

Hence we may define the 2-transform $Y_{2}(y, \omega)$ of $X(x, \omega)$. We obtain

$$
\begin{aligned}
\Delta_{\varepsilon}^{2} Y_{2}(-\varepsilon, \omega) & =Y_{2}(\varepsilon, \omega)-2 Y_{2}(0, \omega)+Y_{2}(-\varepsilon, \omega) \\
& =\frac{1}{\sqrt{ }(2 \pi)} \int_{-\infty}^{\infty} X(x, \omega) \frac{4 \sin ^{2}(\varepsilon x / 2)}{x^{2}} d x .
\end{aligned}
$$

In view of (10.16), we have

$$
\lim _{\varepsilon \rightarrow 0} \frac{1}{\varepsilon \sqrt{ }(2 \pi)} \Delta_{\varepsilon}^{2} Y_{2}(-\varepsilon, \omega)=M_{1}(\omega) .
$$

In other words, we get

THEOREM 10.3. Let us suppose that

$$
\frac{1}{T S} \int_{0}^{T} \int_{0}^{S} \rho(x, y) d x d y
$$

converges as $S \rightarrow \infty, T \rightarrow \infty$ and as $S \rightarrow-\infty, T \rightarrow \infty$ and (10.17) is true for every pair of real numbers $S$ and $T$. Then 
(10.18) $\lim _{T \rightarrow \infty} \frac{1}{2 T} \int_{-T}^{T} X(x, \omega) d x=\lim _{\varepsilon \rightarrow 0} \frac{1}{\varepsilon \sqrt{ }(2 \pi)}\left[Y_{2}(\varepsilon, \omega)-2 Y_{2}(0, \omega)+Y_{2}(-\varepsilon, \omega)\right]$,

where $Y_{2}(y, \omega)$ is the 2-transform of $X(x, \omega)$ which exists and (10.18) means that both isides exist and are equal to each other.

\section{BIBLIOGRAPHY}

1. S. Bochner, Harmonic analysis and the theory of probability, Univ. California Press, Berkeley, Calif., 1955.

2. - Lectures on Fourier integrals, Annals of Mathematics No. 42, Princeton Univ. Press, Princeton, N.J., 1959.

3. S. Bochner and S. Izumi, Some general convergence theorems, Tôhoku Math. J. 42 (1936), 191-194.

4. H. Cramér, On some classes of non-stationary stochastic processes, Proc. 4th Berkeley, Sympos. Math. Statist . and Prob., Vol. II, Univ. California Press, Berkeley, Calif., 1961, pp. 57-78.

5. J. L. Doob, Stochastic processes, Wiley, New York, 1953.

6. H. Hatori, Some expansion theorems for stochastic processes. I, Kōdai Math. Sem. Rep. 15 (1963), 111-120.

7. T. Kawata, Some convergence theorems for stationary stochastic processes, Ann. Math. Statist. 30 (1959), 1192-1214.

8. M. Loève, Fonctions aléatoires du second ordre, Suppl. to P. Lévy, Processus stochastique et mouvement brownien, Gauthier-Villars, Paris, 1948.

9. - Probability theory, 3rd ed. Van Nostrand, Princeton, N.J., 1963.

10. E. Parzen, An approach to time series analysis, Ann. Math. Statist. 32 (1961), 951-989.

CATHOLIC University OF America,

WASHINGTON, D. C. 